\section{Prevalência da adesão ao tratamento anti-hipertensivo em hipertensos resistentes e validação de três métodos indiretos de avaliação da adesão}

\author{
Prevalence of anti-hypertensive treatment \\ adherence in patients with resistant hypertension \\ and validation of three indirect methods for \\ assessing treatment adherence
}

Katia Vergetti Bloch 1

André Nascimento de Melo 1

Armando R. Nogueira 2

\footnotetext{
${ }^{1}$ Instituto de Estudos em Saúde Coletiva, Universidade Federal do Rio de Janeiro, Rio de Janeiro, Brasil. 2 Hospital Universitário Clementino Fraga Filho Universidade Federal do Rio de Janeiro, Rio de Janeiro, Brasil.

Correspondência K. V. Bloch

Instituto de Estudos em Saúde Coletiva, Universidade Federal do Rio de Janeiro. Rua Eurico Cruz 47, apto. 501, Rio de Janeiro, $R$ 22461-200, Brasil.

kbloch@globo.com
} 
ços especializados, sendo importante a distinção entre pressão não-controlada por não-adesão ao tratamento e resistência ao controle da pressão arterial 7 .

Um paciente é considerado aderente quando toma $80-110 \%$ dos medicamentos, pois verificouse que acima de $80 \%$ a pressão arterial diastólica foi adequadamente controlada 8 .

A avaliação da adesão ao tratamento farmacológico não é uma tarefa fácil. Cada método de quantificação da adesão (diretos e indiretos) descrito na literatura tem suas limitações, sem haver um método ideal, cuja sensibilidade e especificidade sejam superiores a $80 \% 9$. A contagem de pílulas, utilizada como padrão-ouro em alguns estudos 2, requer a distribuição integral da medicação pela unidade de saúde, o que geralmente não corresponde à realidade da maior parte dos hipertensos, principalmente dos resistentes, em uso de mais de três drogas, muitas não padronizadas no Sistema Único de Saúde.

O objetivo primário deste estudo foi estimar a prevalência de adesão à terapêutica anti-hipertensiva em hipertensos resistentes empregando-se diferentes métodos. Secundariamente foi realizada a validação preditiva desses métodos utilizando-se a redução da pressão arterial de consultório e a monitorização ambulatorial da pressão arterial (MAPA).

\section{Métodos}

Trata-se de um estudo para estimar a prevalência de adesão ao tratamento anti-hipertensivo em uma coorte de hipertensos resistentes de um hospital universitário. O tamanho amostral ( $\mathrm{n}=210$ ) baseou-se em uma estimativa de adesão de $50 \%$, um erro $\alpha=5 \%$, precisão $=5 \%$ e $10 \%$ de perdas. Foram selecionados os 210 primeiros pacientes em tratamento há pelo menos seis meses, que realizaram ao menos uma MAPA e que vieram à consulta entre janeiro e maio de 2004.

Os métodos de avaliação da adesão utilizados foram:

- Pelo paciente: o paciente atribuía uma nota à sua adesão utilizando uma escala visual de 0 a 5 , ( 1 = não toma ou raramente, 2 = às vezes toma, $3=$ toma quase sempre, $4=$ toma a maioria das vezes, $5=$ toma sempre). A nota 5 o classificava como aderente.

- Pelo médico: atribuição de uma nota de 0 a 5 à adesão do paciente. Escala semelhante à apresentada ao paciente.

- Teste de Morisky-Green (TMG) 10 adaptado para a língua portuguesa 11: 1 - você às vezes tem problemas em se lembrar de tomar a sua medicação? 2 - você às vezes se descuida de tomar seu medicamento? 3 - quando está se sentindo melhor, você às vezes pára de tomar seu medicamento? 4 - às vezes, se você se sentir pior ao tomar a medicação, você pára de tomá-la? O paciente é considerado aderente quando responde não para todas as perguntas.

A forma de classificação da adesão utilizada nos três instrumentos seguiu o padrão que considera aderente quem toma $80 \%$ ou mais da medicação prescrita 8 .

A prevalência da adesão e seu respectivo intervalo de 95\% de confiança (IC95\%) foram estimados segundo três estratégias: (i) específica por método; (ii) concordância dos três métodos ("todos"); (iii) por pelo menos um dos três métodos ("qualquer").

A medida da pressão arterial de consultório e a MAPA foram realizadas seguindo-se as recomendações de diretrizes 1,3 . Utilizou-se esfigmomanômetros de coluna de mercúrio com manguitos de tamanhos apropriados e a MAPA foi realizada com aparelho oscilométrico DYNAMAPA (equipamentos Mobil O Graph, versão 12, Cardios, São Paulo, Brasil). A leitura da pressão arterial foi feita a cada 10 minutos durante o dia e a cada 20 minutos durante a noite. A Figura 1 mostra os momentos nos quais foram realizadas as medidas analisadas.

Na validação preditiva da adesão utilizou-se três medidas para comparar os níveis tensionais de pacientes classificados como aderentes/nãoaderentes aplicando-se o teste Kruskal-Wallis:

- Médias das pressões arteriais sistólicas/diastólicas no momento da avaliação da adesão $\left(\mathrm{T}_{3}\right)$; - Médias de redução entre as duas medidas de pressão arterial de consultório (PA2-PA1);

- Médias de redução entre as duas MAPAs (MAPA2-MAPA1) realizadas com intervalo médio de um ano.

Foram utilizadas médias e diferenças médias, pois nessa população o controle da pressão arterial $(<140 / 90 \mathrm{mmHg})$ é difícil; por isto não foi analisada a acurácia dos testes.

O estudo foi aprovado por comitê de ética em pesquisa e o os pacientes assinaram termo de consentimento informado.

\section{Resultados}

Dos 229 pacientes elegíveis para participar do estudo foram entrevistados 200 atendidos no programa de hipertensão. As características da população e as prevalências de adesão são apresentadas na Tabela 1. Quando se considera os três métodos de adesão ao tratamento conjuntamente, $11,9 \%$ dos pacientes foram classificados como não-aderentes. 


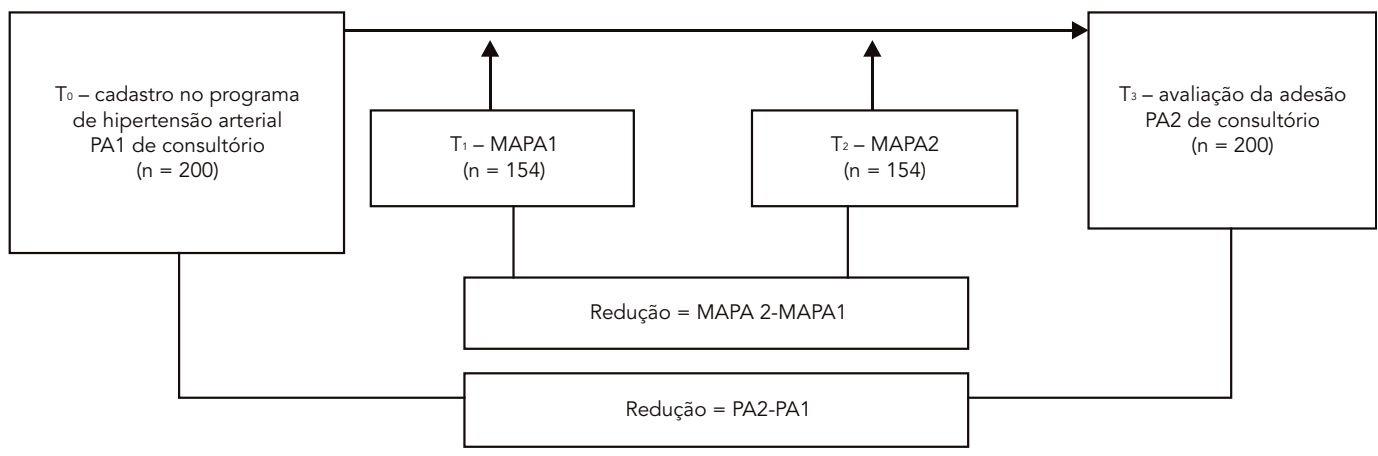

PA: pressão arterial; MAPA: monitorização ambulatorial da pressão arterial.

Tabela 1

Características da população e prevalências de adesão.

\section{Características}

\begin{tabular}{|c|c|}
\hline Idade média em anos (DP) & $63(10,3)$ \\
\hline Mínima-máxima & $38-87$ \\
\hline Sexo feminino & $73,50 \%$ \\
\hline \multicolumn{2}{|l|}{ Escolaridade } \\
\hline Nível fundamental incompleto & $72,70 \%$ \\
\hline \multicolumn{2}{|l|}{ Faixas de IMC } \\
\hline Sobrepeso $\left(25,0-29,9 \mathrm{~kg} / \mathrm{m}^{2}\right)$ & $36,20 \%$ \\
\hline Obesidade grau I $\left(30,0-34,9 \mathrm{~kg} / \mathrm{m}^{2}\right)$ & $28,60 \%$ \\
\hline Obesidade grau II $\left(35,0-39,9 \mathrm{~kg} / \mathrm{m}^{2}\right)$ & $15,30 \%$ \\
\hline Obesidade grau III ( $\left.\geq 40,0 \mathrm{~kg} / \mathrm{m}^{2}\right)$ & $5,60 \%$ \\
\hline Número médio de anti-hipertensivos (DP) & $3,9(0,9)$ \\
\hline Mínimo-máximo & $3 /$ jun \\
\hline \multicolumn{2}{|l|}{ Esquemas terapêuticos } \\
\hline Diurético + IECA + beta-bloqueador & $19,00 \%$ \\
\hline Diurético + IECA + beta-bloqueador + bloqueador de canais de cálcio & $18,50 \%$ \\
\hline \multicolumn{2}{|l|}{ Prevalências de adesão (IC95\%) } \\
\hline Teste de Morisky-Green & $51,0 \%(43,9-58,1)$ \\
\hline Avaliação do médico & $52,0 \%(44,4-59,1)$ \\
\hline Avaliação do paciente & $80,5 \%(74,3-85,8)$ \\
\hline Três métodos positivos & $29,4 \%(22,8-36,7)$ \\
\hline Qualquer dos métodos positivos & $88,1 \%(82,4-92,5)$ \\
\hline
\end{tabular}

DP: desvio-padrão; IMC: índice de massa corporal; IECA: inibidor da enzima conversora da angiotensina.

Independente do método utilizado, as médias da pressão arterial de consultório no momento da avaliação da adesão $\left(\mathrm{T}_{3}\right)$ foram maiores nos pacientes não-aderentes. As diferenças foram maiores quando a adesão foi avaliada pelo médico ou por "qualquer" método, para pressões arteriais sistólicas e diastólicas (Tabela 2). 
Pressão de consultório no momento da avaliação da adesão e médias de diferenças da pressão arterial por cada método.

\begin{tabular}{|c|c|c|c|c|c|c|c|c|c|c|c|c|c|c|c|}
\hline & \multicolumn{15}{|c|}{ Pressão arterial $(\mathrm{mmHg})$} \\
\hline & \multicolumn{3}{|c|}{$\begin{array}{l}\text { Adesão pelo teste de } \\
\text { Morisky-Green (TMG) }\end{array}$} & \multicolumn{3}{|c|}{$\begin{array}{l}\text { Adesão pela nota } \\
\text { do médico }\end{array}$} & \multicolumn{3}{|c|}{$\begin{array}{c}\text { Adesão pela nota } \\
\text { do paciente }\end{array}$} & \multicolumn{3}{|c|}{$\begin{array}{l}\text { Adesão por "todos" } \\
\text { os métodos }\end{array}$} & \multicolumn{3}{|c|}{$\begin{array}{c}\text { Adesão por "qualquer" } \\
\text { dos métodos }\end{array}$} \\
\hline & Sim & Não & $\begin{array}{l}\text { Valor } \\
\text { de } p\end{array}$ & Sim & Não & $\begin{array}{l}\text { Valor } \\
\text { de } p\end{array}$ & Sim & Não & $\begin{array}{l}\text { Valor } \\
\text { de p }\end{array}$ & Sim & Não & $\begin{array}{l}\text { Valor } \\
\text { de } p\end{array}$ & Sim & Não & $\begin{array}{l}\text { Valor } \\
\text { de p }\end{array}$ \\
\hline \multicolumn{16}{|l|}{ Na avaliação } \\
\hline \multicolumn{16}{|l|}{ da adesão } \\
\hline PAS consultório & 156,1 & 160,0 & 0,39 & 151,9 & 166,5 & $<0,01$ & 156,8 & 163,0 & 0,31 & 154,8 & 160,7 & 0,21 & 156,7 & 175,3 & $<0,01$ \\
\hline PAD consultório & 86,2 & 91,4 & 0,02 & 85,1 & 92,9 & $<0,01$ & 87,7 & 93,2 & 0,08 & 84,6 & 90,6 & 0,01 & 87,7 & 97,4 & 0,02 \\
\hline \multicolumn{16}{|l|}{$\begin{array}{l}\text { Médias de } \\
\text { diferenças * }\end{array}$} \\
\hline PAS consultório & 24,2 & 18,0 & 0,11 & 22,6 & 19,2 & 0,57 & 21,8 & 18,5 & 0,39 & 21,5 & 20,7 & 0,51 & 22,6 & 9,0 & 0,10 \\
\hline PAD consultório & 14,1 & 11,5 & 0,11 & 14,5 & 11,1 & 0,10 & 13,9 & 8,2 & 0,10 & 14,4 & 12,2 & 0,14 & 13,6 & 7,3 & 0,10 \\
\hline PAS vigília & 4,3 & $-0,5$ & 0,14 & 5,9 & $-2,6$ & $<0,01$ & 4,0 & $-6,0$ & 0,02 & 3,5 & 1,0 & 0,27 & 3,9 & $-13,0$ & $<0,01$ \\
\hline PAD vigília & 3,0 & $-0,8$ & 0,05 & 3,0 & $-1,0$ & 0,03 & 2,4 & $-3,7$ & $<0,01$ & 2,9 & 0,2 & 0,13 & 2,2 & $-7,5$ & $<0,01$ \\
\hline PAS 24 horas & 4,0 & $-0,5$ & 0,14 & 5,5 & $-2,4$ & $<0,01$ & 3,8 & $-6,0$ & 0,03 & 3,7 & 0,8 & 0,24 & 3,6 & $-12,2$ & $<0,01$ \\
\hline PAD 24 horas & 2,3 & $-1,1$ & 0,05 & 2,3 & $-1,3$ & 0,04 & 1,8 & $-3,6$ & 0,02 & 2,5 & $-0,2$ & 0,13 & 1,7 & $-7,2$ & $<0,01$ \\
\hline
\end{tabular}

* De consultório: PA2-PA1; MAPA: MAPA2-MAPA1.

PAS: pressão arterial sistólica; PAD: pressão arterial diastólica.

Nos pacientes com adesão por "qualquer" método ocorreu redução das pressões arteriais de consultório e na MAPA, enquanto que para os pacientes sem adesão a redução foi menor ou inexistente. As diferenças observadas pelas demais classificações foram menores.

\section{Discussão}

A prevalência da adesão usando-se o TMG foi semelhante à descrita na literatura internacional 10, porém, maior do que a encontrada em estudos nacionais 2,12 realizados com hipertensos menos graves e sem adaptação do instrumento.

Não temos conhecimento de outros estudos que tenham avaliado a adesão de forma sistemática em hipertensos resistentes. Um estudo de hipertensos resistentes acompanhados em hospital terciário americano mostrou que apenas $16 \%$ dos pacientes referiram não ser aderentes, mas a forma de avaliação da adesão não foi apresentada 13 .

A prevalência de adesão ao se considerar o conjunto dos três métodos foi elevada, o que pode ser devido ao acompanhamento em um programa específico para hipertensos resistentes. Embora a restrita generalização possa ser considerada uma limitação do estudo, o dado sobre adesão é fundamental para caracterizar a hiper- tensão resistente e pouco investigado de forma pragmática 7 .

Pacientes com maior adesão apresentaram menores níveis tensionais e maiores reduções da pressão arterial. Prado et al. ${ }^{2}$ encontraram associação entre controle da pressão arterial e adesão, e também entre o TMG e a contagem de pílulas, utilizando pressão de consultório.

A pressão arterial de consultório foi maior nos pacientes sem adesão do que nos com adesão. As reduções das pressões arteriais de vigília e de 24 horas permitiram uma melhor avaliação da adesão do que a redução da pressão arterial de consultório. A vantagem da MAPAé a ausência do efeito do jaleco branco, que poderia mascarar a redução da pressão dos pacientes com adesão, além de ser mais significativa por representar a média de diferentes medidas no período e não uma medida casual 14 .

O TMG não apresentou um bom desempenho isoladamente. Outros estudos não encontraram associação entre o TMG e controle da pressão arterial em hipertensos menos graves 2,12.

O emprego de mais de um método para avaliação da adesão mostrou que indivíduos classificados como não-aderentes pelos três métodos tiveram pior evolução dos níveis tensionais e, portanto, permite a identificação na prática clínica de indivíduos que se beneficiariam de estratégias para aumento da adesão. Os resultados 
encontrados deixam claro que a resistência ao tratamento anti-hipertensivo não deve ser atribuída primariamente ao não-controle da hipertensão arterial por baixa adesão ou tratamento inadequado, uma vez que a prevalência de nãoadesão, utilizando-se três métodos indiretos de avaliação, foi baixa em uma população acompanhada em um serviço de referência.

\section{Resumo}

O estudo estimou a adesão ao tratamento anti-hipertensivo farmacológico utilizando-se três métodos indiretos em uma coorte de hipertensos resistentes no Rio de Janeiro, Brasil, 2005. Os métodos foram: avaliação pelo paciente; avaliação do médico; teste de MoriskyGreen (TMG) adaptado para a lingua portuguesa. Foi realizada validação preditiva comparando-se a diferença tanto de pressões de consultório como de monitorização de 24 horas (MAPA), em duas ocasiões, de pacientes com e sem adesão. As médias de pressões entre os grupos foram comparadas usando-se testes não-paramétricos. Foram entrevistados 200 pacientes com idade média de 63 anos (DP = 10,3), 73,5\% do sexo feminino. A prevalência de adesão foi de $51 \%$ pelo TMG, 52\% pelo médico e $80,5 \%$ pelo paciente. Ocorreram reduções das pressões arteriais de consultório e na MAPA dos pacientes com adesão por todos os métodos, mas não para os não-aderentes. O emprego de mais de um método para avaliação da adesão mostrou que indivíduos não-aderentes pelos três métodos $(11,9 \%)$ tiveram pior evolução dos níveis tensionais. Esse achado sugere que a hipertensão resistente não pode ser atribuída unicamente à baixa adesão.

Hipertensão; Validade dos Testes; Assistência Ambulatorial

\section{Colaboradores}

K.V. Bloch participou do planejamento do estudo e análise, discussão, redação e revisão do artigo. A. N. Melo participou do planejamento do estudo, coleta de dados e análise, discussão e redação do artigo. A. R. Nogueira participou da discussão e redação do artigo. 


\section{Referências}

1. V Diretrizes Brasileiras de Hipertensão Arterial. Arq Bras Cardiol 2007; 89:e24-79.

2. Prado JC, Kupek E, Mion D. Validity of four indirect methods to measure adherence in primary care hypertensives. J Hum Hypertens 2007; 21:579-84.

3. The Seventh Report of the Joint National Committee on Prevention, Detection, Evaluation and Treatment of High Blood Pressure. JAMA 2003; 289:2560-72.

4. McAlister FA, Lewanczuk RZ, Koon KT. Resistant hypertension: an overview. Can J Cardiol 1996; 12:822-8.

5. Thrift AG, McNeil JJ, Forbes A, Donnan GA. Three important subgroups of hypertensive persons at greater risk of intracerebral hemorrhage. Hypertension 1998; 31:1223-9.

6. Lessa I. Impacto social da não-adesão ao tratamento da hipertensão arterial. Rev Bras Hipertens 2006; 13:39-46.

7. Calhoun DA, Jones D, Textor S, Goff DC, Murphy TP, Toto RD, et al. Resistant hypertension: diagnosis, evaluation, and treatment a scientific statement from the American Heart Association Professional Education Committee of the Council for High Blood Pressure Research. Hypertension 2008; 51:1403-19.

8. Sackett DL, Snow JC. The magnitude of compliance and noncompliance. In: Haynes RB, Taylor DW, Sackett DL, editors. Compliance in health care. Baltimore: John Hopkins University Press; 1979. p. 11-22.
9. Piñeiro F, Gil V, Donis M, Orozco D, Torres MT, Merino I. Validez de 6 métodos indirectos para valorar el cumplimiento del tratamiento farmacológico en las dislipemias. Aten Primaria 1997; 19:465-8.

10. Morisky DE, Green LW, Levine DM. Concurrent and predictive validity of a self-reported measure of medication adherence. Med Care 1986; 24: 67-74.

11. Melo NA. Avaliação da adesão à terapêutica antihipertensiva em pacientes hipertensos resistentes [Dissertação de Mestrado]. Rio de Janeiro: Instituto de Estudos em Saúde Coletiva. Universidade Federal do Rio de Janeiro; 2005.

12. Strelec MAAM, Pierin AMG, Mion Jr. D. The influence of patient's consciousness regarding high blood pressure and patient's attitude in face of disease controlling medicine intake. Arq Bras Cardiol 2003; 81:349-54.

13. Garg JP, Elliott WJ, Folker A, Izhar M, Black HR. Hypertension service. Resistant hypertension revisited: a comparison of 2 university-based cohorts. Am J Hypertens 2005; 18:619-26.

14. Verdecchia P. Prognostic value of ambulatory blood pressure: current evidence and clinical implications. Hypertension 2000; 35:844-51.

Recebido em 09/Abr/2008

Versão final reapresentada em 18/Jul/2008 Aprovado em 12/Ago/2008 